\title{
Stochastic Modelling to Enable Ultrafast High Resolution Capacitive Position Sensing of a Resonant MEMS Mirror
}

\author{
Lisa-Marie Faller ${ }^{1}$ and Hubert Zangl ${ }^{1}$ \\ 1 Institute of Smart System Technologies, \\ Sensors and Actuators, \\ Universitätsstrasse 65-67, 9020 Klagenfurt, Austria \\ Lisa-Marie.Faller@aau.at
}

\begin{abstract}
:
With the increased interest in adoption of rapid prototyping processes for high throughput manufacturing lines, uncertainties in topology and physics arise. These uncertainties cannot be captured anymore by conventional simulation techniques without major efforts. Thus grows the necessity to compensate adverse effects on the side of signal processing rather than in the sensor front end. This can be done utilizing models which incorporate available prior knowledge. It is shown that utilizing a model for the motion of a resonant MEMS mirror, the uncertainty of the position estimate can be reduced to a level which would otherwise be prohibited by thermal noise. To meet the requirements with respect to update rate and latency, it is mandatory that the algorithm can be implemented in hardware. This is done in an FPGA platform with a specifically designed analogue front-end.
\end{abstract}

Key words: Capacitive sensing, Extended Kalman filtering, Noise Analysis, Microelectromechanical Systems (MEMS), Sensor modelling, Sensor simulation.

\section{Introduction}

The employment of MicroElectroMechanical Systems (MEMS) is common practice nowadays. Among such systems, optical MEMS, such as resonating mirrors, facilitate lightweight spectrometers and other handheld optical measurement equipment. Formerly, such equipment used to be bulky and expensive. On the one hand, MEMS mirrors provide a convenient way to decrease size and costs while at the same time enabling faster measurement rates. On the other hand, such innovations do not only provide advantages, they also present research and industry with new challenges: the smaller the equipment, the smaller the

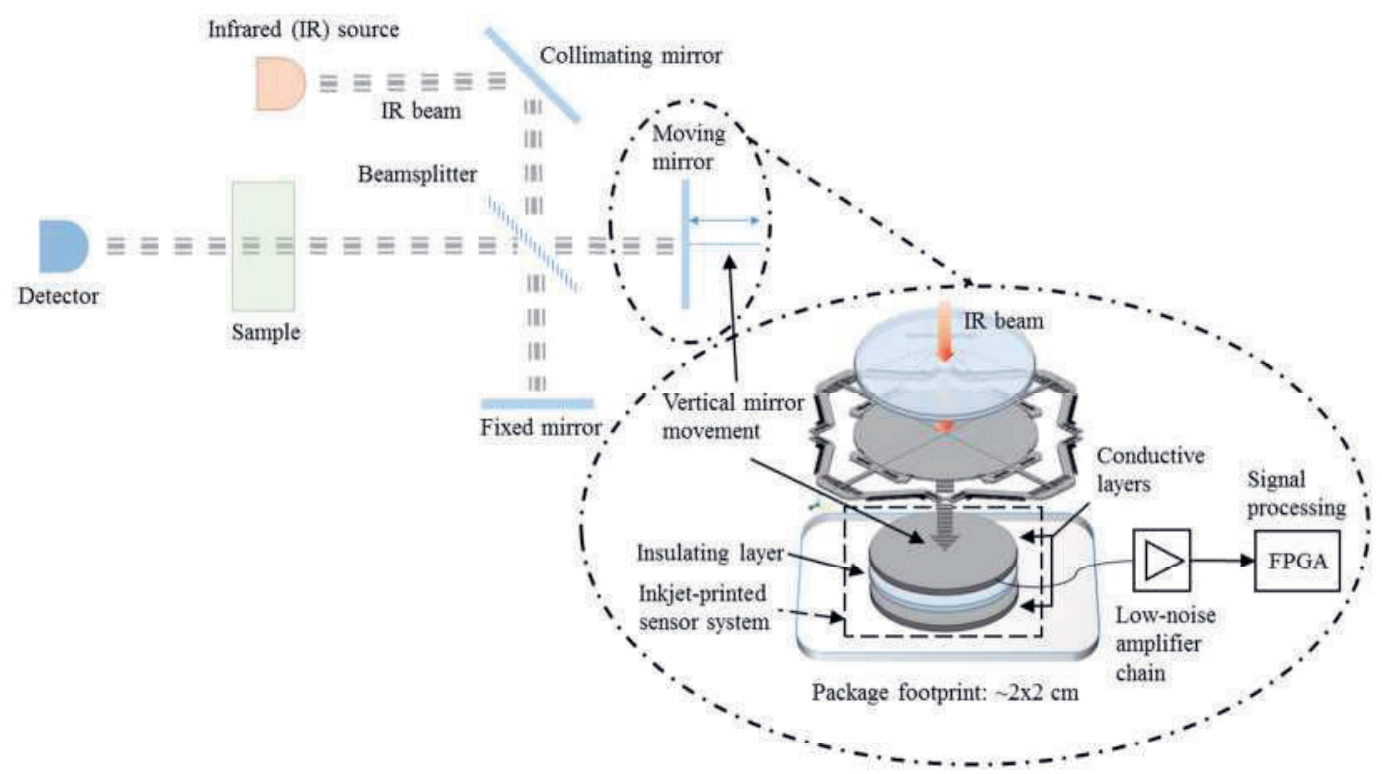

Fig. 1: Basic construction principle of a Michelson interferometer in the upper left. On the lower right is the MEMS mirror, the inkjet-printed capacitive sensor system: sensor front-end, Low-Noise Amplifiers (LNA) and signal processing hardware. 
components need to be. This, as well, is true for transduction mechanisms and reference measurement setups contained in the miniaturized equipment. Additionally, devices built with cutting-edge technology oftentimes demand individually adaptable solutions and designs.

To serve these demands in the considered Fourier Transform InfraRed spectrometer (FTIR) as illustrated in Fig.1, a capacitive position sensing front-end for the moving mirror is suggested. It is comparably easy to manufacture and integrate in a rapid prototyping manner (e.g. inkjet-printed electrodes). Additionally, the capacitive sensing principle is generally capable of providing high resolutions and high measurement rates.

The position measurement system for the moving mirror is the benchmarking element for the spectrometer resolution. It is therefore subject to concise requirements. Relevant figures are: a wide measurement range of $r_{m}=$ $1000 \mu \mathrm{m}$ at $\mathrm{d}_{0}=1000 \mu \mathrm{m}$ offset and a required position resolution of respos $=50 \mu \mathrm{m}$.

The inkjet-printed front-end and resonant MEMS mirror provide for geometrical and topological uncertainties and deficiencies. Consequently, we show that intelligent system modelling and signal processing can provide a solution and enable high accuracy when physical system properties are hard to control.

\section{Interferometer and Sensing System}

A common way to manufacture an FTIR spectrometer device is combining an infrared light source with a Michelson interferometer setup (shown in Fig. 1). In this setup, two mirrors are employed which are suspended in an angle of $90^{\circ}$. One of these mirrors is moveable to generate interference patterns (see [1] for details). One movement over the full possible pathlength of the moving mirror is called a scan. To improve the Signal-to-Noise Ratio (SNR), an average over multiple scans is used. The resulting interference pattern can then be used to identify substances of interest (Sample in Fig.1). To keep the measurement time low and provide sufficient SNR, fast position tracking of the moving mirror's position is as necessary as to add the gathered patterns congruently.

\section{MEMS Mirror}

The considered mirror (see Fig. 2) is manufactured in a CMOS compatible SiliconOn-Insulator (SOI) technology. The functional, highly p-doped, silicon device layer is $75 \mu \mathrm{m}$ on a wafer of $600 \mu \mathrm{m}$ thickness. The final coating is $50 \mathrm{~nm}$ of aluminium to generate a reflective surface [2]. The suspension of this device relies on four pantographs and is specifically designed to suppress parasitic modes. Ideally, an absolute mirror movement of $1000 \mu \mathrm{m}$ at a resonance frequency of $500 \mathrm{~Hz}$ is possible. To achieve maximum pathlengths, the mirror package is evacuated down to $50 \mathrm{~Pa}$ pressure. The considered devices were reported to reach a spectral resolution of res $_{\mathrm{spec}}=25 \mathrm{~cm}^{-1}$ at $\mathrm{d}_{\mathrm{tr}}=$ $200 \mu \mathrm{m}$ translatory displacement with an acquisition time of $t_{a q}=200 \mu s$ per scan [3].

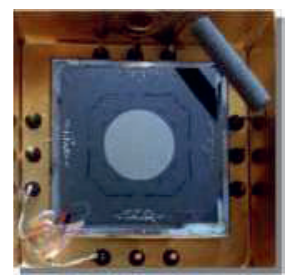

Fig. 2. MEMS- mirror device (light grey circle) assemble in vacuum-ready package with vacuum sensor and ion getter.

\section{State of the Art: Capacitive Sensing}

The application of a micro-manufactured capacitive sensor, realized as multilayer structure ( $1 \mathrm{~mm}$ below the mirror plate), comes with the advantage of a slim and readily integrable sensing solution while providing for high resolution measurement capabilities, compare [4], [5].

Capacitive sensors can be designed to work in either single-ended or differential mode. In both modes, the change in capacitance between conductive surfaces (electrodes), at different electric potentials, is measured. Applying a voltage to one electrode, the transmitter, and measuring the resulting displacement current in a second electrode, i.e. the receiver, is termed differential mode. In a single-ended design, the displacement current at the transmitter is determined. Measurements in single-ended mode are possible to the open environment or distant ground.

The capacitive sensor will be manufactured as flat electrode structure. It then forms a parallel plate capacitor with the mirror plane, which is a common setup for nanometer applications [6]. The sensor is thus comparatively simpler to realize and integrate into an existing package than is the reference interferometer setup. A capacitive sensor, manufactured by a rapid prototyping technology, can be advantageous compared to other manufacturing strategies (e.g. [7], [8] and [9]) since it enables individual designs and can be applied to various setups.

The read-out of capacitive sensors is commonly done using, e.g., Capacitance to Digital Converters (CDCs). Improvements on CDCs have been presented in, e.g. [10]. Commercially available CDCs provide, e.g., res $s_{p o s}=100 \mathrm{~nm}$ 
equivalent position resolution at an update rate of $f s=90 \mathrm{~Hz}$ [11]. Off-the-shelf systems are also available: the capaNCDT by $\mu \varepsilon$ [12] provides res $_{\text {pos }}=40 \mathrm{~nm}$ and $\mathrm{a}$ bandwidth of $B=8.25 \mathrm{kHz}$. The Microsense 8810 system [13] can provide res $s_{\text {pos }}=5 \mathrm{~nm}$ at a rate of $B=1 \mathrm{kHz}$. The D-510 system provided by Physical Instruments is designed for a distance offset $d_{0}=750 \mu \mathrm{m}$ and is thus not fully comparable. The Microsense, $\mu \varepsilon$ and PI Systems cannot be integrated into the MEMS package outline. Measurement ranges and average sensor distances of the considered devices are also smaller than required. The aforementioned systems measure capacitance directly. Alternatively, capacitance measurements can also be performed using a shunt. This concept is used for the system we suggest, as well as in the HF2 by Zurich Instruments. The HF2 employs lock-in amplifiers to realize the amplification of the sensor signal. Though the HF2 is comparable in terms of bandwidth, the reported input referred noise figure of $\left(u_{n}=5 \mathrm{nV} / \mathrm{NHz}\right)$ limits the position resolution to, in our setup, respos $=120 \mathrm{~nm}$. A comparison of available devices (see also [14]) is provided in Fig. 3: to improve readability, the bandwidth of the target system in the illustration is lower $(B=60 \mathrm{kHz})$ than the true specified bandwidth of up to $B=180 \mathrm{MHz}$. Also for the HF2, the bandwidth in the illustration $(B=20$ $\mathrm{kHz}$ ) is lower than the true bandwidth of up to $B$ $=50 \mathrm{MHz}$. Most of the presented systems available up to now, provide sufficiently low noise by considering small bandwidths. Systems operating at higher bandwidth suffer from higher measurement noise due to thermal noise power integrated over the considered bandwidth. Additionally, all the presented systems lack the possibility to access and adapt the signal processing directly. Thus, the small necessary latency cannot be reached by any of those hardware platforms

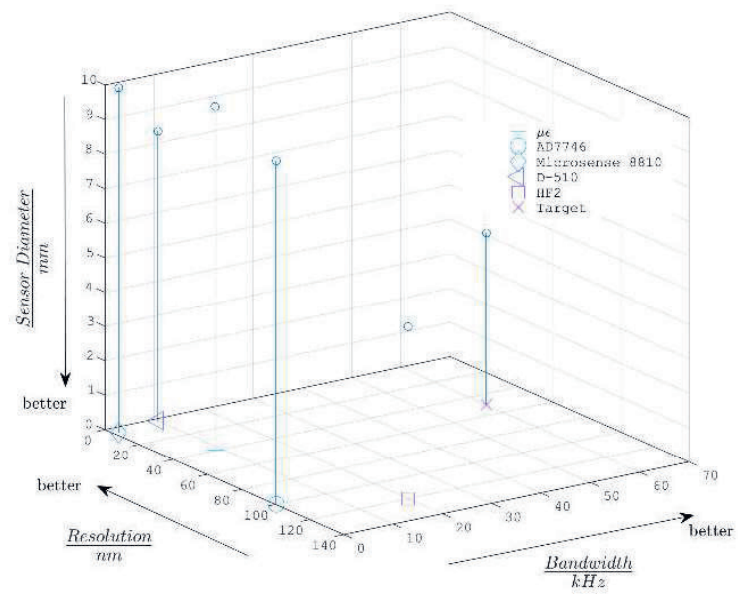

Fig. 3 Graphical comparison of available capacitive position sensing systems and the target device.

\section{Simulations}

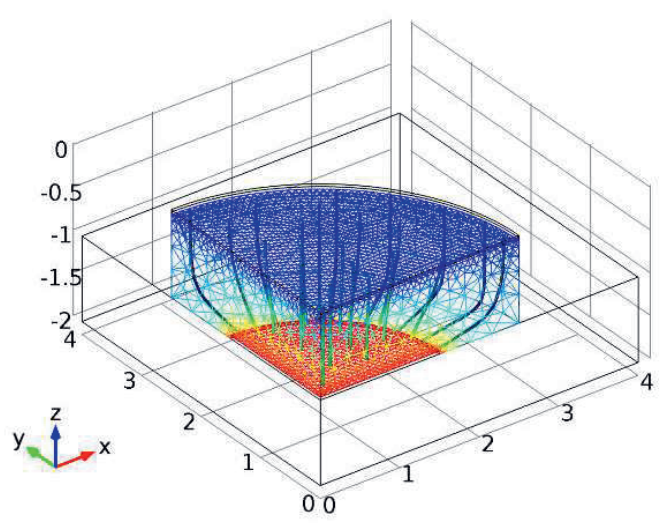

Fig. 4 Simulation setup where one quarter of the mirror geometry is sufficient due to symmetry reasons.

Finite Element Method (FEM) simulations (compare also [15]) are set up to consider all effective influences (e.g. fringing fields etc.). In Fig. 4, a simplified simulation setup is shown: The electrostatic field evolution is illustrated as coloured tubes emanating from the sensing electrode surface (red) and terminating at the mirror plane (blue). The mirror movement is implemented by a moving mesh feature. The movement of the geometry is known, so the displacement of the mesh can be calculated at every time step using e.g. a Laplace smoothing approach [16]. After the mesh deformation is determined, the electrostatics are computed by solving Gauss law

$$
\nabla D=\rho_{V}
$$

where the electric displacement field is

$$
D=\varepsilon_{0} E
$$

and $\rho_{V}$ is the space charge density. This way, the capacitance to distance relation can be determined.

\section{Sensor Modelling}

In a first approach to a suitable semi-analytic model for the capacitive position sensor, a polynomial model is fitted to data from FEM simulations. This is a commonly used process and well-documented in literature [17], [18]. We presume, the behaviour of the measured signal, i.e. the capacitance, $C\left(d_{m}\right)$ with $d_{m}$ the parameter of interest, i.e. the mirror position, can be described as inversely proportional to each other. The fitted non-linear regression model is of the form

$$
\mathrm{C}\left(\mathrm{d}_{m}\right)=A_{1} \frac{1}{\mathrm{~d}_{m}}+A_{2} \mathrm{~d}_{m}+A_{3}
$$

Here, also other transformations of the distance are possible. The coefficients $A_{1}$ to $A_{3}$ are 
determined using a Least Squares Estimator (LSE).

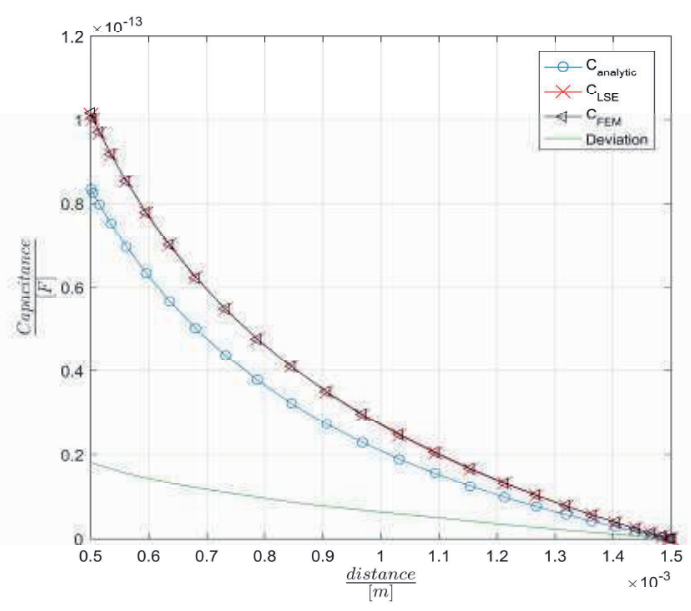

Fig. 5 Graphical comparison of the analytically determined capacitance $C_{\text {analytic, }}$ the capacitance determined from the simulation $C_{F E M}$ (and fitted curve $\left.C_{L S E}\right)$ and their deviation from each other.

\section{Results}

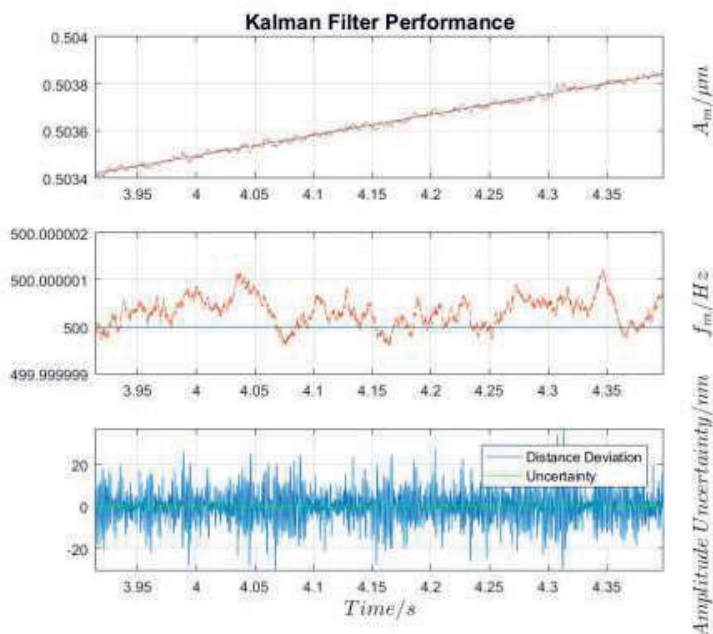

Fig. 6 Result of one evaluation of the EKF yielding the estimated amplitude $A_{m}$ and frequency $f_{m}$ (red curves) versus true values (blue curves) in the first two plots. The lowermost plot illustrates the achieved distance deviation in blue and the minimum uncertainty in green.

The determined model is then used as basis of an Extended Kalman Filter (EKF) as already addressed in [19]. Evaluations of the EKF using the metamodel for the measurement function show a minimum achievable position error of $\sim 20 \mathrm{~nm}$

\section{Conclusion}

We developed a sensor model for a capacitive inkjet-printed sensor based on FEM simulations. The simulations consider also specific material and geometry properties of the device. By means of simulation and proper modelling, we build the theoretical foundation of the suggested measurement system. Thus, we demonstrated the feasibility of a high-resolution measurement system without the need for sophisticated frontend optimization.

\section{Acknowledgement}

This project has been supported by the COMET K1 ASSIC Austrian Smart Systems Integration Research Center. The COMET-Competence Centers for Excellent Technologies Program is supported by BMVIT, BMWFW and the federal provinces of Carinthia and Styria.

\section{References}

[1] B. C. Smith, Fundamentals of Fourier Transform Infrared Spectroscopy, CRC Press, 2011

[2] T. Sandner, T. Grasshoff, and H. Schenk, Translatory MEMS actuator with Extraordinary Large Stroke for Optical Path Length Modulation, IEEE International Conference on Optical MEMS \& Nanophotonics.

[3] H. Schenk, P. Dürr, T. Haase, D. Kunze, U. Sobe, H. Lakner H. and Kück, Large Deflection Micromechanical Scanning Mirrors for Linear Scans and Pattern Generation, IEEE Journal of Selected Topics in Quantum Electronics, 6(5).

[4] M. Carminati, G. Ferrari, F. Guagliardo, and M. Sampietro, "ZeptoFarad Capacitance Detection with a Miniaturized CMOS Current Front-End for Nanoscale Sensors," Sensors and Actuators, A: Physical, 2011.

[5] T. Zeng, Y. Lu, Y. Liu, H. Yang, Y. Bai, P. Hu, Z. $\mathrm{Li}$, and J. Tan, "A Capacitive Sensor for the Measurement of Departure from the Vertical Movement," IEEE Transactions on Instrumentation and Measurement, vol. 65, no. 2, 2016.

[6] T. Hicks, P. Atherton, The NanoPositioning Book, Queensgate Instruments Limited, 1997.

[7] S. I. Moore, S. O. R. Moheimani, Displacement Measurement with a Self-Sensing MEMS Electrostatic Drive, J. of Microelectromech. Syst. 23 (3) (2014) 511-513.

[8] M. S.-C. Lu, G. K. Fedder, Position Control of Parallel-Plate Microactuators for Probe-Based Data Strorage, J. of Microelectromech. Syst. 13 (5) (2004) 759-769.

[9] B. Cagdaser, B. E. Boser, Low-Voltage Electrostatic Actuation With Inherent Position Feedback, J. of Microelectromech. Syst. 21 (5) (2012) 1187-1196.

[10] V. Sreenath, B. George, An Easy-to-Interface CDC With an Efficient Automatic Calibration, IEEE Trans. on Instrumentation and Measurement 65 (5) (2016) 960-967.

[11] Analog Devices (Feb. 2016). [link]. URL http://www.analog.com/media/en/technicaldocumentation/data-sheets/AD7745 7746. 
[12] Microepsilon (June 2016). [link]. URL http://www.micro-epsilon.com/displacementposition-sensors/capacitivesensor/capaNCDT 6500/index.html.

[13] Microsense (June 2016). [link]. URL http://www.microsense.net/products-positionsensors-microsense-8800.htm.

[14] A. J. Fleming, K. K. Leang, Design, Modeling and Control of Nanopositioning Systems, Springer International Publishing Switzerland, 2014.

[15] L.-M. Faller, H. Zangl, Robust Design of a 3Dand Inkjet-Printed Capacitive Force/Pressure Sensor, IEEE Proceedings of the EuroSimE, 2016.
[16] Comsol multiphysics documentation, version 5.2 (Feb. 2016).

[17] A. J. Fleming, "A Review of Nanometer Resolution Position Sensors: Operation and Performance," Sensors and Actuators, A: Physical, vol.190, pp. 106-126, February 2013.

[18] T. Hicks and P. Atherton, The NanoPositioning Book. Queensgate Instruments Limited, 1997.

[19] L.-M. Faller and H.Zangl, Feasibility Considerations on an Inkjet-Printed Capacitive Sensor for Electrostatically Actuated Resonant MEMS Mirror Systems, J. of Microelectromech. Syst. pp (99) (2017) 1-10. 